\title{
Study of the interplay between magnetic shear and resonances using Hamiltonian models for the magnetic field lines
}

\author{
M.-C. Firpo, ${ }^{1}$ and D. Constantinescu ${ }^{2}$ \\ ${ }^{1}$ Laboratoire de Physique des Plasmas, CNRS - Ecole Polytechnique, 91128 Palaiseau cedex, France \\ ${ }^{2}$ Dept of Applied Mathematics, Association Euratom-MECI, \\ University of Craiova, Craiova 200585, Romania
}

\begin{abstract}
The issue of magnetic confinement in magnetic fusion devices is addressed within a purely magnetic approach. Using some Hamiltonian models for the magnetic field lines, the dual impact of low magnetic shear is shown in a unified way. Away from resonances, it induces a drastic enhancement of magnetic confinement that favors robust internal transport barriers (ITBs) and stochastic transport reduction. When low-shear occurs for values of the winding of the magnetic field lines close to low-order rationals, the amplitude thresholds of the resonant modes that break internal transport barriers by allowing a radial stochastic transport of the magnetic field lines may be quite low. The approach can be applied to assess the robustness versus magnetic perturbations of general (almost) integrable magnetic steady states, including non-axisymmetric ones such as the important single helicity steady states. This analysis puts a constraint on the tolerable mode amplitudes compatible with ITBs and may be proposed as a possible explanation of diverse experimental and numerical signatures of their collapses.
\end{abstract}

PACS numbers: 52.55.Fa,05.45.+a,52.25.Gj

\section{INTRODUCTION}

In the present hot plasma toroidal devices, it is widely recognized that the essential limitation that prevents ignition to be reached is due to an insufficient energy confinement resulting from small-scale turbulence. During the last two decades, some decisive progress has however been made to increase the energy confinement time through the widespread experimental achievement of internal transport barriers (ITBs) [1, 2]. In these regimes, there exists a region of the tokamak plasma core where the local ion and electron thermal diffusivities are substantially reduced, nearly down to their neoclassical values [2]. This is likely to be a signature of the regularity of the central magnetic surfaces. In this way, the emergence of ITBs results in a sort of virtuous circle for fusion with both high central temperature $T$ and high energy confinement time $\tau_{E}$ increasing the triple product $n_{e} T \tau_{E}$, which implies a better fusion yield. The importance of the magnetic shear, through the form of safety factor profiles, in reaching these states by quenching the core plasma turbulent transport has been firmly established. Decisive ingredients were given notably by the experimental results obtained by Eriksson et al. [3] who demonstrated experimentally that the $q$-profile could be used as a single control parameter to obtain ITBs. In these experiments, they could obtain ITBs for non-monotonic $q$-profiles but not with monotonously growing $q$, while other parameters such as the $\mathbf{E} \times \mathbf{B}$ flow shear were kept the same. Additional evidence was later given by Sauter et al. [4] through a dedicated experiment. They controlled the $q$-profile using inductive current to generate positive and negative current density perturbations in the plasma center, with negligible input power. In this way, they were able to demonstrate that the electron confinement could be modified significantly solely by perturbing the current density profile. Other experiments in various tokamaks showed that having an inner region with low magnetic shear was indeed sufficient to obtain ITBs [2, 5].

In reverse field pinches (RFPs) also, improved performance related to magnetic chaos healing [6] has been recently achieved experimentally, yet through non-axisymmetric states [7 9 . It was observed that, by increasing the plasma current, the RFP operated some change of magnetic topology through a spontaneous transition towards a singlehelicity equilibrium strongly resilient to magnetic chaos.

These various experimental results emphasize the importance of the magnetic structure as they show that some configurations - reversed-shear, or low-shear, $q$-profiles in tokamaks or some single-helicity equilibria in RFPs - enable a substantial improvement of the confinement. This is an invitation to consider a purely magnetic approach of the problem [10] that will be pursued here. This amounts to address the issue of the plasma confinement in terms of the magnetic confinement, namely in terms of the confinement of the magnetic field lines. The ensuing approach is thus obviously a simplified approximation of a more complex, self-consistent, reality involving a larger number of effective

\footnotetext{
*Electronic address: marie-christine.firpo@lpp.polytechnique.fr
} 
degrees of freedom, including in particular the electric field. It is a sort of zero order approach that relies on the essential principle of magnetic confinement devices which is to use the magnetic field to confine the charged particles composing the fusion fuel in a plasma state [11]. In this sense, ensuring the confinement of the magnetic field lines may be viewed as a prerequisite to particle and energy confinement. As it is well known, this purely magnetic approach may be addressed within an Hamiltonian formalism that results from the universal divergence-free nature of $\mathbf{B}$ [12]. An intermediate, first-order, step towards a fully realistic description of the physics of magnetic confinement devices would be to treat the test particle motion [13] instead of the magnetic field lines. This is left for a future work.

In the absence of resonances, that will be specified below, the dramatic improvement on magnetic confinement induced by a low magnetic shear condition will be recalled in Section II and its link to ITBs and core stochastic transport reduction discussed. The improved confinement observed in RFPs in single-helicity states will be shown to result from the same mechanism when moving to appropriate action-angle variables. However this optimistic scenario will be tempered since, in actual tokamaks, ITBs may eventually collapse. In Section III, it will be shown, using some Hamiltonian models for the magnetic field lines, that this low shear condition happens to be strongly deleterious to magnetic confinement when approaching resonances by enabling stochasticity for much lower magnetic perturbation amplitudes. This will be further analyzed in Section IV. A discussion of this phenomenon and of its possible consequences will be finally given in Section $\mathrm{V}$.

\section{BENEFITS OF A LOW MAGNETIC SHEAR}

\section{A. Hamiltonian formulation for the magnetic field lines}

It is well known that the equations of magnetic field lines can be cast in Hamiltonian form. Actually, the field lines of a magnetic, $\mathbf{B}(\mathbf{x})$, or any other divergence-free field are the trajectories of a Hamiltonian (See e.g. the review by Boozer [12] or Abdullaev's book [26]).

In tokamaks, a dependance on the toroidal angle breaking the rotational invariance is analogous to the usual timedependence in Hamiltonian dynamical systems. Generically one is thus left with a one-and-a-half degrees of freedom Hamiltonian, that can be decomposed into an axisymmetric, integrable part and a perturbation term that may be Fourier decomposed in the poloidal and toroidal angles. For tokamaks, the Hamiltonian reads

$$
H(\psi, \theta, \phi)=H_{0}(\psi)+\sum_{m, n} H_{m n}(\psi) \cos \left(m \theta-n \phi+\chi_{m n}\right),
$$

with $H_{0}(\psi)=\int^{\psi} W\left(\psi^{\prime}\right) d \psi^{\prime}$ and $W(\psi)=1 / q(\psi)$ the winding profile, inverse of the safety factor profile. In the tokamak configuration, $H$ is the poloidal magnetic flux and $\psi$ the toroidal magnetic flux conjugated to the poloidal angle $\theta$.

\section{B. Some results on the benefits of low shear}

In real magnetic fusion devices, the magnetic perturbations given by the wave amplitudes $H_{m n}(\psi)$ may be induced by a variety of phenomena. They may result from external effects, such as the ripple, or may result from intrinsic phenomena such as MHD activity. In order to address the magnetic confinement problem, one may turn to simplified models for the form of the perturbed magnetic field. Restricting to the single poloidal number $m=1$, taking equal phases $\chi_{1 n}$ and some special form of the radial perturbation $H_{1 n}(\psi)$ identical for all $n$ 's yields the continuous version of the tokamap [14]. Then, considering the limit of a large number of toroidal modes makes the associated Poincaré (toka)map appear naturally through the use of periodic $\delta$ distributions.

Using the symmetric tokamap framework, it was recently shown in Ref. [10] that the existence of a low magnetic shear region produced a drastic enhancement of the confinement of the magnetic field lines. More specifically, the existence of some $\psi_{s}$ such that $H_{0}^{\prime \prime}\left(\psi_{s}\right)=0$, that amounts to $W^{\prime}\left(\psi_{s}\right)=0$, was shown to improve significantly the robustness w.r.t. magnetic perturbations of the regular core magnetic field lines inside the $\psi=\psi_{s}$ surface, acting then as a transport barrier, in agreement with previous results obtained in so-called non-KAM systems including mainly maps with reversed shear profiles (See e.g. [15-20]). Interestingly, Ref. [10] gave evidence that this effect does not necessitate $q$ to be reversed shear: the $q$-profile can also be monotonous with an inflexion point, which agrees with the diversity of ITBs experimental scenarios. The beneficial effect of the radial extent of the low shear zone for safety profiles with $q>1$ was demonstrated. Recently, these results were more rigorously grounded: Theorems of existence of invariant circles for non-twist maps were formulated for the symmetric tokamap [21] using a theorem about small shear systems formulated by Ortega [22]. 


\section{Extension to non-axisymmetric states}

It is important to note that the domain of applicability of this favorable regime may be extended from axisymmetric equilibria to non-axisymmetric integrable states provided $\psi$ is replaced by some suitable action variable. Let us consider the case where some magnetic configuration involves not only an axisymmetric component $H_{0}(\psi)$ but also some $\left(m_{0}, n_{0}\right)$ component whose amplitude $A$ is much larger than that of the other $(m, n)$ waves of order $\varepsilon$. The Hamiltonian reads then

$$
H(\psi, \theta, \phi)=H_{0}(\psi)+A H_{m_{0} n_{0}}(\psi) \cos \left(m_{0} \theta-n_{0} \phi\right),
$$

up to $\mathcal{O}(\varepsilon)$ terms. The dominant part of the Hamiltonian, given in Eq. (2), may be put into an explicitly integrable form

$$
\bar{H}(\Psi, \Theta)=H_{0}\left(m_{0} \Psi\right)-n_{0} \Psi+A H_{m_{0} n_{0}}\left(m_{0} \Psi\right) \cos \Theta
$$

through a canonical change of variables, with the generating function $F_{2}(\Psi, \theta, \phi)=\left(m_{0} \theta-n_{0} \phi\right) \Psi$, that amounts to moving to the $\left(m_{0}, n_{0}\right)$ wave frame. Even if $H_{0}^{\prime \prime}(\psi)$ does not vanish, it is then easy to recognize that, provided $A$ is large enough, a shearless condition in the action variable

$$
J \equiv \frac{1}{2 \pi} \oint \Psi d \Theta
$$

associated to the integrable part of the Hamiltonian (2) may however be obtained. In this way, there can be nonaxisymmetric magnetic states that are much more resilient to magnetic chaos than what would be their axisymmetric components. An illustration of this phenomenon was given in Ref. [6]. It was shown that the magnetic structure involving some small $m=1$ magnetic island with a separatrix was less robust towards magnetic perturbations than a reconnected structure with a higher $m=1$ amplitude. This transition in terms of the winding profile in the action variable $J$ may be interpreted as follows: In the low amplitude case where a magnetic island is present, the separatrix is associated to a vanishing of $W(J)$ within a cusp singularity, whereas, as the amplitude grows, the reconnection process eventually makes the separatrix disappears inducing locally the growth of $W(J)$ and smoothing the singularity, previously associated to the separatrix, which causes the appearance of a local minimum of $W(J)$. This later favorable process may be responsible of the unexpectedly good confinement of the non-axisymmetric self-organized states very recently achieved in a RFP [7] and in a compact toroidal [23] plasmas. For the tokamak setting, this non-axisymmetric formulation of the low shear or zero-twist condition may open new perspectives to improve magnetic confinement.

\section{DETRIMENTAL EFFECT OF LOW SHEAR IN THE VICINITY OF RESONANCES USING SOME HAMILTONIAN MODELS}

Let us now go back to the notations introduced in Eq. (11). The above analysis did not take into account the possibility of resonances. In particular, the fact that the winding number $W_{s} \equiv W\left(\psi_{s}\right)$, associated to the action $\psi_{s}$ such that $H_{0}^{\prime \prime}\left(\psi_{s}\right)=0$, could be in the vicinity of some phase velocity $n / m$ corresponding to the $(m, n)$ magnetic perturbation was not considered. For instance, works using the tokamap framework [10, 14, 24] ignored de facto this possibility by restricting to the $m=1$ case while taking typically $q$-profiles above 1 (with the noticeable exception of the sawtooth-related studies of Ref. [25]).

In order to illustrate the interplay between low shear and resonances, let us first consider a simplification of the system (1) similar to the continuous tokamap case but where only the $m=4$ poloidal modes are retained, with

$$
H(\psi, \theta, \phi)=\int^{\psi} \frac{d x}{q(x)}+K \widehat{H}(\psi) \sum_{n=-M}^{M} \cos (4 \theta-n \phi) .
$$

For $M \geq 1$, this represents a one-and-a-half degrees of freedom Hamiltonian in which the perturbation amounts to a superposition of plane waves with winding numbers equal to $n / 4$. It is convenient, and physically relevant, to consider the large $M$ case for which the derivation of the symplectic mapping associated to (5) is immediate through the use of the periodic- $\delta$ distribution. For computational purposes, we used a symmetric version [18, 26] of the later mapping that yields a very accurate modeling of the continuous dynamics (5) with $\widehat{H}(\psi)=2 \psi /(1+\psi)$. The physical motivation to consider this specific form of magnetic perturbation was discussed in Ref. [14]. Basically, this form roughly mimics the radial (or $\psi$ ) form of MHD tearing modes. It gives a vanishing perturbation in the limit $\psi \rightarrow 0$ 
and ensures that the polar axis cannot be crossed so that, under the map, $\psi$ remains positive as it should be. It would have been possible to use fully realistic MHD profiles for each $(m, n)$ mode obtained from experiments (as e.g. in the study of Ref. [27]). Our point in the present Section is however to use a simpler framework in order to emphasize the specific role of $W_{s}$.

In order to use $W_{s}$ as a single control parameter, we considered the family of winding profiles $W_{p}(\psi)=W_{s}-c\left(\psi-\psi_{s}\right)^{2}$ with fixed $c$ and $\psi_{s}$. These correspond to reversed shear $q$-profiles with zero shear at $\psi_{s}$. To be specific, $W_{s}$ was allowed to vary while putting $c=.5$ and $\psi_{s}=0.4$ in the whole study.

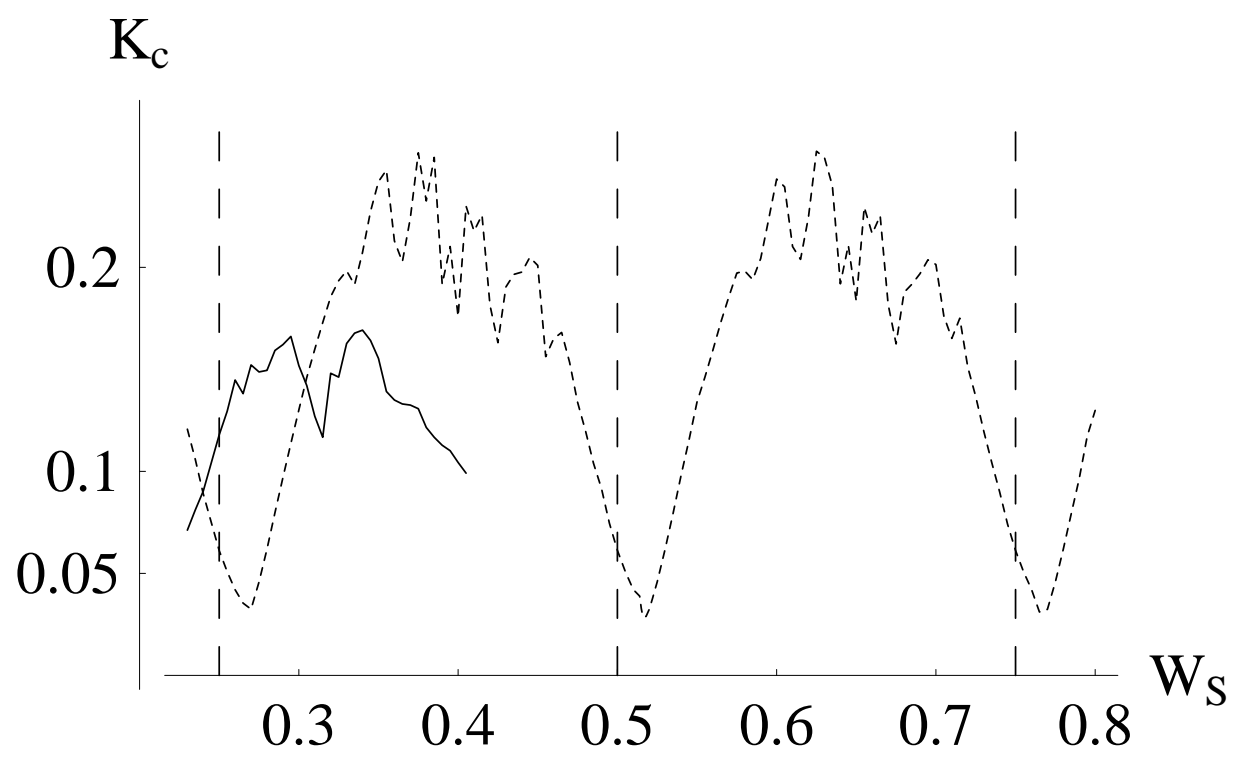

FIG. 1: The critical value of the stochasticity parameter $K$, as defined in the text, as a function of the inverse of the minimum of the $q$-profile, $W_{s}$, for the parabolic family $W_{p}(\psi)$ (dashed line) and as a function of the parameter $W_{s}$ in the linear case $W_{l}(\psi)$ (plain line). The vertical dashed lines mark the locations of the resonances $W_{s}=1 / 4,2 / 4$ and $3 / 4$.

In order to quantify the impact of the specific value of the minimum of the $q$-profile, $q_{\min }$, (or of its inverse $\left.W_{s}=1 / q_{\min }\right)$, we defined by $K_{c}$ the value of the stochasticity parameter $K$ above which there exists some initial condition with $\psi<0.1$ whose evolution under (5) eventually escapes the physically accessible phase space by crossing $\psi=1$. Practically, for each value of $W_{s}$ and a given value of $K$, up to one hundred initial conditions were chosen at random in the range $0<\psi<0.1$ and allowed to evolve under the symmetric map obtained from Eq. (5) for up to 5000 iterations. If some $\psi$ happened to cross $\psi=1$, the process was relaunched for a smaller $K$ or else, for a larger $K$, in a dichotomic way, so as to converge towards the percolation-like threshold $K_{c}$ value. The obtained results have been plotted in Fig. 11. Let us first briefly comment on the meaning of the indicator $K_{c}$. Using the form of the magnetic perturbations as $\widehat{H}(\psi)=2 \psi /(1+\psi)$ ensures that core magnetic field lines remain regular whereas border field lines (at $\psi=1)$ are the first to feel chaos as $K$ increases. Having in mind fusion applications, $K_{c}$ has a practical meaning in terms of a measure of the confinement of the magnetic field lines, as it gives a threshold above which magnetic field lines starting from the core eventually reach the border. It is also an indicator of the global quantitative measure of the stochasticity of the magnetic field lines since, in the present model, there cannot be a connection between the core and the border of the $\psi$-domain through a regular magnetic field trajectory. However, with respect to the magnetic confinement issue, $K_{c}$ may underestimate chaos, in the sense that there may exist non-negligible subsets of the physical central phase space domain in which chaotic field lines are chaotic already for values of $K$ below $K_{c}$.

It is straightforward to realize that, for the profiles $W_{p}(\psi)$ or $W_{l}(\psi) \equiv W_{s}-c\left(\psi-\psi_{s}\right)$, the dynamics is invariant under a translation in $W_{s}$ of some multiple of $1 / 4$. Effectively the curve giving $K_{c}$ as a function of $W_{s}$ is $1 / 4$-periodic in $W_{s}$ up to statistical discrepancies. Some part of the curve obtained for the linear strong shear case $W_{l}(\psi)$ has been drawn for comparison for about one period in $W_{s}$. Basically, the effect of low shear happens to be efficient for $W_{s}$ between the $n / 4$ resonances by increasing significantly $K_{c}$ w.r.t. the high shear case. The Poincaré plot of Figure 2 corresponds to such a favorable situation. This reflects the fact that, in spite of a non-negligible perturbation of magnitude $K$, winding profiles having a low shear region can sustain almost regular core magnetic surfaces. This magnetic chaos healing is likely to explain the stochastic transport reduction in ITBs due to the sole effect of the $q$-profile reported in Refs. [3, 4]. 


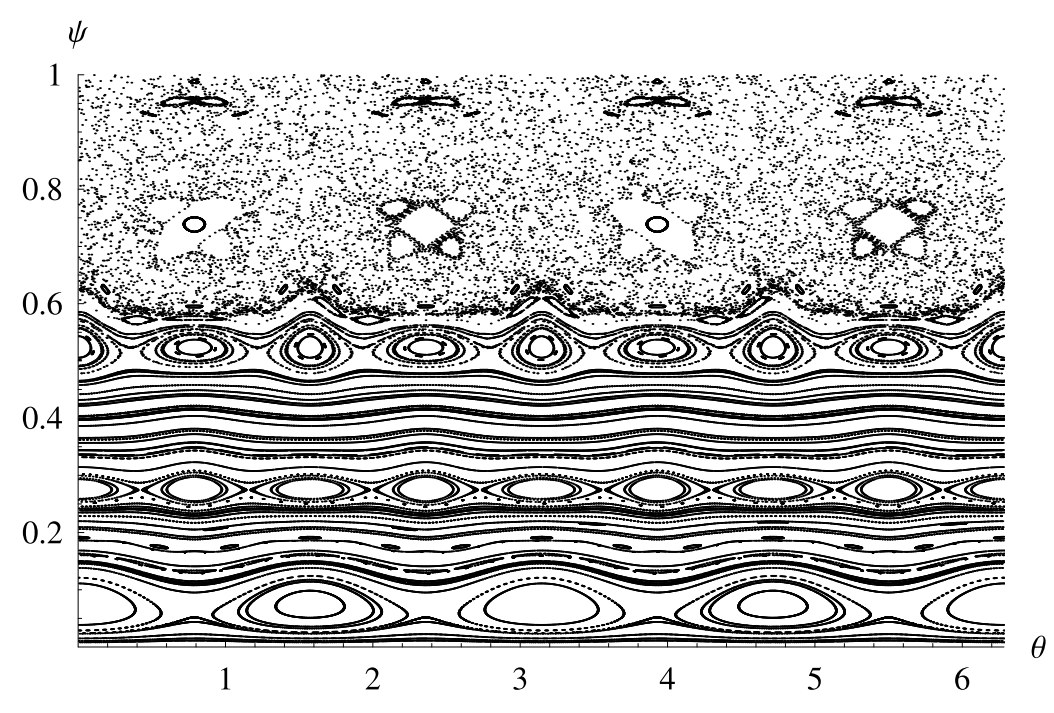

FIG. 2: Poincaré plot of the system (5) with the parabolic reversed-shear profile $W_{p}(\psi)$ for $K=0.03$ and $W_{s}=0.645$.

However, in the vicinity of resonances, this tendency reverses: low shear induces abrupt drops of the magnetic confinement whereas quite higher threshold values of $K$ are reached for the high-shear linear winding profile. In this case, the statement formulated by Rosenbluth, Sagdeev, Taylor and Zaslavski [28] that strong shear is beneficial to preserve the regularity of the magnetic surfaces applies.

For the reversed-shear winding profile $W_{p}(\psi)$, low minima of $K_{c}$ occur for values of $W_{s}$ slightly above the linear values of the resonances given by $n / 4$. Figure 3 depicts what happens in this case. The abrupt degradation of magnetic confinement in the vicinity of the resonances coincides with the emergence of some local stochastic web pattern [29 31] that enables a large diffusion in the action $\psi$. Under the perturbation, separatrices of the island chains, a characteristic phase-space pattern of the non-twist Hamiltonian systems, are destroyed and replaced with stochastic channels of finite width, visible on this Figure. This produces a path to some deleterious radial transport already at low values of the amplitude of the resonant mode. The merging of separatrices is the phenomenon through which global chaos enters the system and will be more closely examined in Section IV] In another physical context, Soskin et al. studied a close phenomenon of nonlinear resonance in the minimal case of a single exciting wave [32]. Here, the consideration of a large mode number $M$, that is physically relevant to account for turbulence in toroidal fusion devices, should provide an additional effective randomization of motion in the vicinity of the separatrix.

All other things being equal, we then considered the Hamiltonian system for the magnetic field lines (5) obtained by just replacing $m=4$ by $m=2$ and computed its associated symmetric map. Figure 4 displays the obtained values of the threshold $K_{c}$ defined for two cases of winding profiles, the parabolic one $W_{p}(\psi)$ and the quadratic one $W_{q}(\psi) \equiv W_{s}-c\left(\psi-\psi_{s}\right)^{4}$, together with the curve previously obtained for $m=4$ for the reversed-shear parabolic profile. This time $K_{c}$ is plotted as a function of the minimum of the safety factor profile $q_{\min } \equiv 1 / W_{s}$, a parameter more commonly used by the tokamak community. In the $m=2$ case, the positive role on magnetic confinement induced by the existence of a low shear region away from resonances appears in full clarity. The threshold $K_{c}$ attains there very large values and the comparison with the $m=4$ case is illuminating: the span in $W_{s}$ between two subsequent $m=2$ resonances is twice larger than the one between two subsequent $m=4$ resonances and $K_{c}$ is then allowed to grow to larger amplitudes between resonances. The effect of the amount of flatness around $q_{\text {min }}$ is investigated by comparing the results obtained for $W_{p}$ to that for the quadratic flattest profile $W_{q}$ in the $m=2$ case. Away from resonances, it is clear that expanding the low shear zone improves the resilience of the magnetic structure towards perturbations as shown in Ref. [10]. However, in the vicinity of resonances, the effect reverses. A lower minima of $K_{c}$ is obtained for $W_{q}$ and it is reached closer to the resonance $q=2 / n$ (with $n=1$ in Fig. (4). 


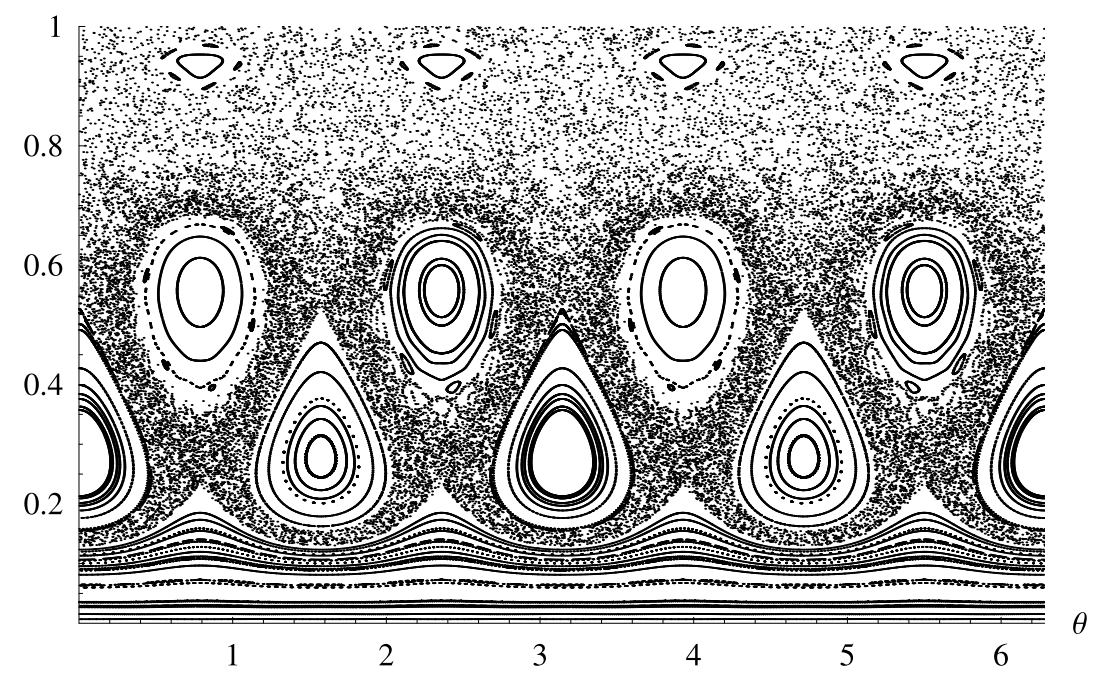

FIG. 3: Poincaré plot of the system (5) with the parabolic reversed-shear profile $W_{p}(\psi)$ for $K=0.03$ and $W_{s}=0.52$.

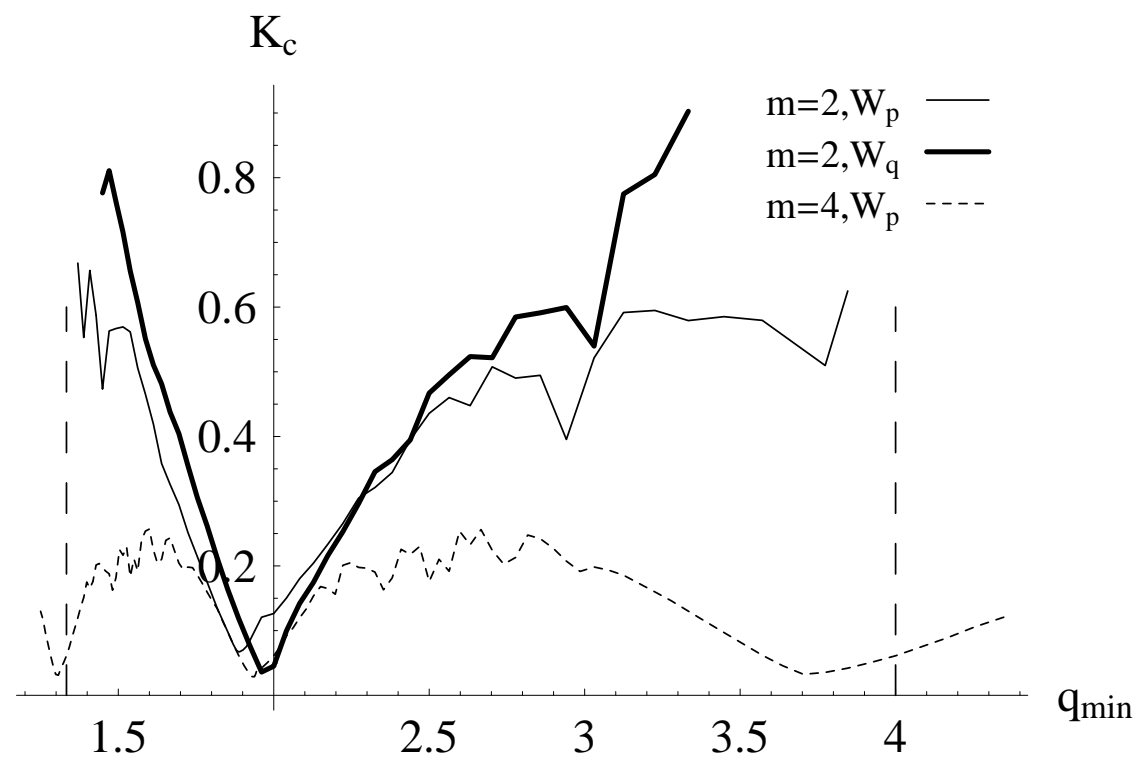

FIG. 4: Critical value of the stochasticity parameter $K$, as defined in the text, as a function of the minimum of the $q$-profile, $q_{\min } \equiv 1 / W_{s}$.

\section{LOCAL ANALYSIS}

We wish to interpret the above results within the continuous Hamiltonian system. Let us consider then the Hamiltonian

$$
H(\psi, \theta, \phi)=\int^{\psi} W(x) d x+K \widehat{H}(\psi) \sum_{n=-M}^{M} \cos \left(m_{0} \theta-n \phi\right)
$$


for some given poloidal mode $m_{0}$ and take $W(\psi)=W_{s}-c\left(\psi-\psi_{s}\right)^{k}$, with $c>0$ and $W_{s}=W\left(\psi_{s}\right)$. Let us proceed to a canonical change of variable with the generating function

$$
F_{2}\left(\psi^{\prime}, \theta, \phi\right)=\left(m_{0} \theta-n_{0} \phi\right) \psi^{\prime}+\psi_{s} \theta
$$

This yields

$$
\begin{aligned}
& \frac{\partial F_{2}}{\partial \theta}=\psi=m_{0} \psi^{\prime}+\psi_{s}, \\
& \frac{\partial F_{2}}{\partial \psi^{\prime}}=\theta^{\prime}=m_{0} \theta-n_{0} \phi
\end{aligned}
$$

and the new Hamiltonian reads, up to a constant,

$$
H\left(\psi^{\prime}, \theta^{\prime}, \phi\right)=\left(m_{0} W_{s}-n_{0}\right) \psi^{\prime}-\frac{c}{k+1}\left(m_{0} \psi^{\prime}\right)^{k+1}+K \widehat{H}\left(m_{0} \psi^{\prime}+\psi_{s}\right) \sum_{n=-M}^{M} \cos \left(\theta^{\prime}+n_{0} \phi-n \phi\right) .
$$

Sufficiently close to the resonance $n=n_{0}, \theta^{\prime}+n_{0} \phi-n \phi$ is slowly varying, so that one can average over the fast "time" $\phi$. This yields the one degree of freedom Hamiltonian

$$
H\left(\psi^{\prime}, \theta^{\prime}, \phi\right)=\left(m_{0} W_{s}-n_{0}\right) \psi^{\prime}-\frac{c}{k+1}\left(m_{0} \psi^{\prime}\right)^{k+1}+K \widehat{H}\left(m_{0} \psi^{\prime}+\psi_{s}\right) \cos \theta^{\prime} .
$$

The study of its topology as a function of $W_{s}$ and $K$ should help to clarify the phase space location where the stochasticity, induced by the perturbation to (11), should first emerge as $K$ increases from zero. Figure 5 shows the
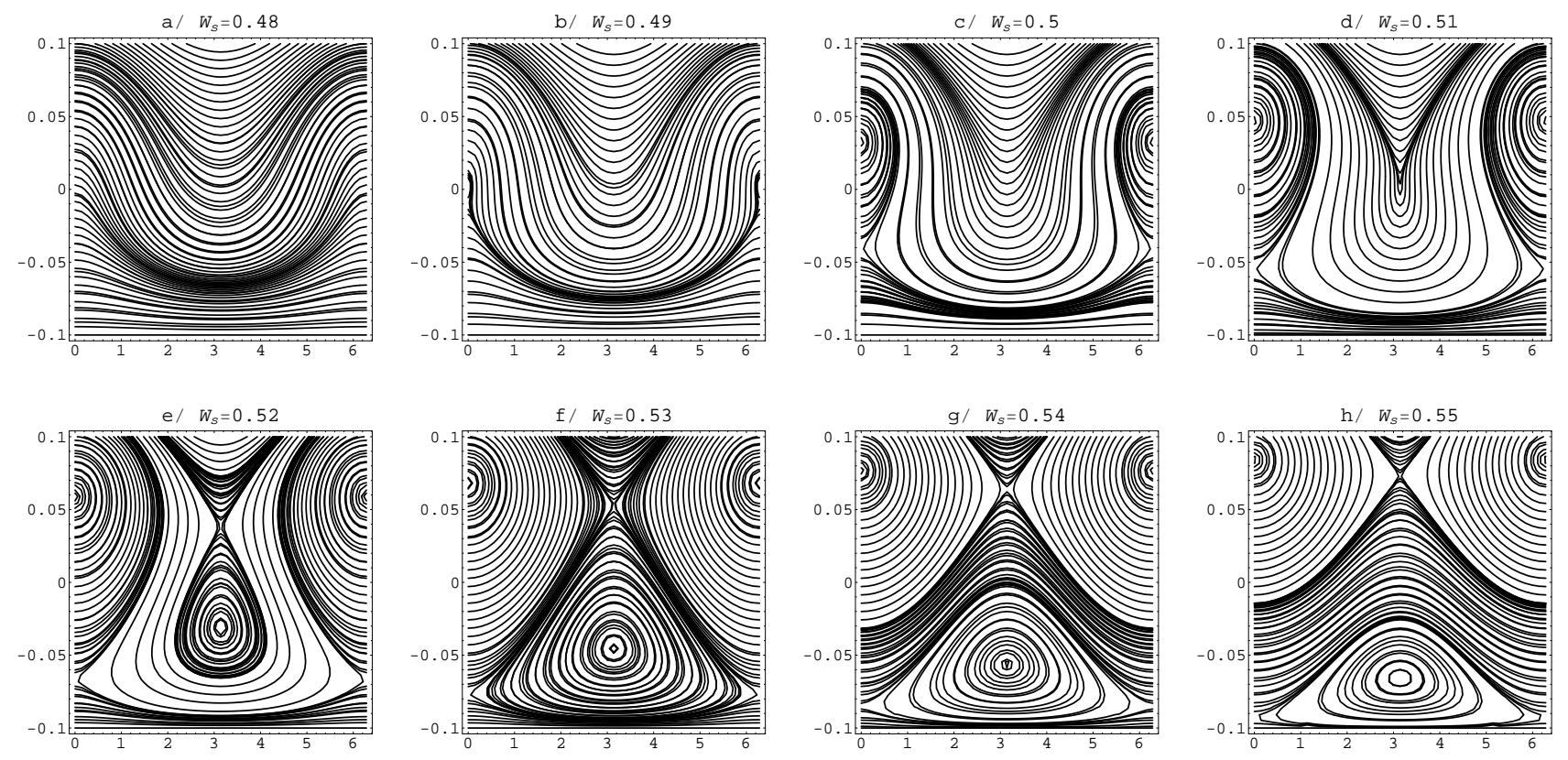

FIG. 5: Phase space of the Hamiltonian (11) for some values of $W_{s}$ close to $n_{0} / m_{0}$ with $m_{0}=4$ and $n_{0}=2$, for $c=0.5$ and $K=0.01$.

transition from an homoclinic topology with dimerized islands (plot e) to an heteroclinic topology with twin islands (plots $\mathrm{g}$ and $\mathrm{h}$ ) as $W_{s}$ increases while being slightly above $n_{0} / m_{0}=1 / 2$. The bifurcation between these two topologies [33] occurs at some reconnection threshold $W_{s}^{*}$ (see e.g. Ref. [34, 35]) that depends on the perturbation amplitude $K$ and can be easily computed by equaling the energy values at the x-points obtained for $\theta=0$ and $\theta=\pi$.

As it is well known, the nonintegrable perturbation coming from the other modes will gradually replace, as $K$ increases, separatrices by stochastic layers. On the basis of Figure 5, one can argue that the largest extent of the stochastic domain shall take place in the case where $W_{s}$ is slightly above $n_{0} / m_{0}$, close to the situation corresponding 
to the plot e, in which case the stochastic layers coming from the two separatrices shall merge for a sufficiently large value of the perturbation amplitude. In this way, this analysis gives account of the fact that the smallest values of the critical thresholds take place, for the given $\psi$-form of the perturbation, not for $W_{s}=n_{0} / m_{0}$ but slightly larger values. The Reader is referred to [26, 36] for extra reference studies on the transition to chaos in Hamiltonian systems with non-monotonic frequency.

\section{DISCUSSION}

The previous qualitative, yet systematic, study has emphasized the dual impact of low magnetic shear on magnetic confinement in toroidal devices. The main achievement of this approach has been to hopefully clarify the dual impact of low magnetic shear through a unified picture. In particular, it has been shown using some Hamiltonian models for the magnetic field lines that, away from any consideration on dynamics and stability, there exists potentially very low thresholds on magnetic perturbations above which safety factor profiles having a low shear region occurring for $q$-values close to $q=m / n$ induce a loss of magnetic confinement. This may now be used both to predict and to interpret experimental observations.

For instance, assume that some reversed-shear $q$-profile associated to an ITB regime evolves in such a way that $q_{\text {min }}$ decreases and crosses e.g. $q=2$. Then, if there exists some magnetohydrodynamic (MHD) mode with $m=2 n$, typically a $(2,1)$ mode, having a sufficiently large amplitude, one predicts a collapse of this ITB due to an abrupt macroscopic degradation of magnetic confinement. This prediction does not need any consideration on the nature of the instability that produced this particular $(2,1)$ mode. However, to make a quantitative statement on the amplitude threshold inducing the collapse one should have some knowledge on the radial form of the modes involved .

This prediction does not either preclude the fact that ITBs may be more easy to trigger experimentally for rational values of $q_{\min }$ for low MHD activity, a fact on which there does not seem to be a general agreement [4] anyway. There is some subtlety here: For instance, for the original symmetric tokamap, that corresponds to the case where only $m=1$ (non-resonant) perturbations are retained, the most robust magnetic surfaces would actually be obtained for $q_{\min }$ close to 2. It could however explain the fact that the good confinement phase obtained in these cases is always transient [37] as various $(m, n)$ modes should eventually be destabilized either linearly or nonlinearly through mode couplings. Moreover, as it is well known from the tokamak linear MHD theory, the low $m$ and $n$ MHD modes are typically the most dangerous ones, in the sense that they are associated to the largest linear growth rates. Consequently, one expects drops of magnetic confinement to take place in the first place for low-order rational values of $q_{\min }$, as the amplitude thresholds requested for the large-scale degradation of the magnetic confinement should be first attained for those low $(m, n)$ modes.

As illustrated in Fig. 4, the negative impact of low shear should be either benign and transient in the case where the $q$-profile happens to rapidly cross low rational values of $q_{\min }$, or particularly deleterious, if the $q$-profile happens to be clamped and very flat about $q_{\min }$ close to some $\bar{q} \equiv m / n$, with a large enough amplitude in the $(\bar{q} n, n)$ modes. These ITBs collapses triggered by stochastization of the magnetic field lines should manifest through flattenings of the pressure and temperature profiles in the stochastic region.

Interestingly enough, these predictions agree with numerous experimental and numerical observations on ITBs dynamics. We should first note that experimental evidences indicate that the so-called Edge-Localized modes (ELMs) may not be responsible for ITBs collapses since these are observed to happen already before the ELMs occur [38]. Additionally, ITB do collapse while the $q$-profile is reversed-shear and there is a variety of experimental report of core confinement collapses in reversed-shear cases when $q_{\text {min }}$ reaches values close to low order rational values such as $3 / 2$, 2 or 3 [38 -40]. The extensive experimental and numerical results of Ref. [41] consistently show that pressure crashes associated to core magnetic stochasticity are strongly correlated with the closeness of $q_{\min }$ to 2 and with a sufficient amplitude in the $(2,1)$ mode. The proposed picture is also consistent with recent numerical results showing that an ITB collapse occurs for $q_{\min }$ in the vicinity of $4 / 3$ when turbulence involves a sufficiently large amplitude of the $(4,3)$ mode [42]. Moreover, the above predictions should be most easily evidenced on electron ITBs, since due to their small Larmor radius, electrons provide a scan of the magnetic field structure. In this respect, it would be interesting to investigate further the connections with the experimental results of Ref. [43] in which the disappearance of electron transport barriers are associated with $q_{\min }$ crossing simple rational values.

Finally, resonances with $m=n$ associated to the $q=1$ surface are by no way special. Sawteeth crashes could then be interpreted as ITB collapses associated to $q=1$. A complete modeling would however require an enlarged framework that is beyond the scope of the present article. 


\section{Acknowledgments}

Comments by A. Vasiliev on stochastic webs are gratefully acknowledged. MCF thanks L. Nasi and J.-M. Rax for useful discussions.

This work was carried out within the framework the European Fusion Development Agreement and the French Research Federation for Fusion Studies. It is supported by the European Communities under the contract of Association between Euratom and CEA. The views and opinions expressed herein do not necessarily reflect those of the European Commission.

[1] R.C. Wolf. Internal transport barriers in tokamak plasmas. Plasma Phys. Control. Fusion, 45:R1-R91, 2003.

[2] J. W. Connor et al. A review of internal transport barriers physics for steady-state operation of tokamaks. Nucl. Fusion, 44:R1-R49, 2004.

[3] L.-G. Eriksson, C. Fourment, V. Fuchs, X. Litaudon, C.D. Challis, F. Crisanti, B. Esposito, X. Garbet, C. Giroud, N. Hawkes, P. Maget, D. Mazon, and G. Tresset. Discharges in the jet tokamak where the safety factor profile is identified as the critical factor for triggering internal transport barriers. Phys. Rev. Lett., 88(145001), 2002.

[4] O. Sauter, S. Coda, T. P. Goodman, M. A. Henderson, R. Behn, A. Bottino, E. Fable, An. Martynov, P. Nikkola, and C. Zucca. Inductive current density perturbations to probe electron internal transport barriers in tokamaks. Phys. Rev. Lett., 94(10):105002, Mar 2005.

[5] K. A. Razumova et al. Formation of electron transport barriers under ecr control of the q(r) profile in the t-10 tokamak. Plasma Phys. Rep., 27:273, 2001.

[6] D. F. Escande, R. Paccagnella, S. Cappello, C. Marchetto, and F. D'Angelo. Chaos healing by separatrix disappearance and quasisingle helicity states of the reversed field pinch. Phys. Rev. Lett., 85(15):3169-3172, Oct 2000.

[7] P. Piovesan D. Terranova P. Zanca M. Zuin A. Alfier D. Bonfiglio F. Bonomo A. Canton S. Cappello L. Carraro R. Cavazzana D. F. Escande A. Fassina P. Franz M. Gobbin P. Innocente L. Marrelli R. Pasqualotto M. E. Puiatti M. Spolaore M. Valisa N. Vianello P. Martin R. Lorenzini, E. Martines, RFX mod team, and collaborators. Self-organized helical equilibria as a new paradigm for ohmically heated fusion plasmas. Nature Physics, 5:570-574, 2009.

[8] M. E. Puiatti, A. Alfier, F. Auriemma, S. Cappello, L. Carraro, R. Cavazzana, S. Dal Bello, A. Fassina, D. F. Escande, P. Franz, M. Gobbin, P. Innocente, R. Lorenzini, L. Marrelli, P. Martin, P. Piovesan, I. Predebon, F. Sattin, G. Spizzo, D. Terranova, M. Valisa, B. Zaniol, L. Zanotto, M. Zuin, M. Agostini, V. Antoni, L. Apolloni, M. Baruzzo, T. Bolzonella, D. Bonfiglio, F. Bonomo, A. Boozer, M. Brombin, A. Canton, R. Delogu, G. De Masi, E. Gaio, E. Gazza, L. Giudicotti, L. Grando, S. C. Guo, G. Manduchi, G. Marchiori, E. Martines, S. Martini, S. Menmuir, B. Momo, M. Moresco, S. Munaretto, L. Novello, R. Paccagnella, R. Pasqualotto, R. Piovan, L. Piron, A. Pizzimenti, N. Pomphrey, P. Scarin, G. Serianni, E. Spada, A. Soppelsa, S. Spagnolo, M. Spolaore, C. Taliercio, N. Vianello, A. Zamengo, and P. Zanca. Helical equilibria and magnetic structures in the reversed field pinch and analogies to the tokamak and stellarator. Plasma Physics and Controlled Fusion, 51(12), DEC 2009. 36th European-Physical-Society Conference on Plasma Physics, Sofia, Bulgaria, Jun 29-Jul 03, 2009.

[9] D. Bonfiglio, M. Veranda, S. Cappello, L. Chacón and G. Spizzo Journal of Physics: Conference Series, 260:012003, 2010.

[10] L. Nasi and M.-C. Firpo. Enhanced confinement with increased extent of the low magnetic shear region in tokamak plasmas. Plasma Phys. Control. Fusion, 51:045006, 2009.

[11] P.J. Morrison Magnetic field lines, Hamiltonian dynamics, and nontwist systems. Phys. Plasmas, 7:2279-2289, 2000.

[12] A. H. Boozer Rev. Mod. Phys., 76: 1071, 2004.

[13] S. S. Abdullaev, A. Wingen, and K. H. Spatschek Mapping of drift surfaces in toroidal systems with chaotic magnetic fields. Phys. Plasmas, 13/042509, 2006.

[14] R. Balescu, M. Vlad, and F. Spineanu. Tokamap : A hamiltonian twist map for magnetic field lines in toroidal geometry. Phys. Rev. E, 58(1):951 - 964, 1998.

[15] D. del Castillo-Negrete, J. M. Greene, and P. J. Morrison. Area preserving nontwist maps: periodic orbits and transition to chaos. Physica D, 91:1-23, 1996.

[16] R. Balescu. Hamiltonian nontwist map for magnetic field lines with locally reversed shear in toroidal geometry. Phys. Rev. E, 58(3):3781-3792, Sep 1998.

[17] D Constantinescu and R Constantinescu. Transport barriers and diffusion phenomena for the magnetic field lines in tokamak. Physica Scripta, 2005(T118):244, 2005.

[18] A. Wingen, K. H. Spatschek, and S. Abdullaev. Stochastic transport of magnetic field lines in the symmetric tokamap. Contributions to Plasma Physics, 45:500513, 2005.

[19] J. S. E. Portela, I. L. Caldas, and R. L. Viana. Tokamak magnetic field lines described by simple maps. The European Physical Journal - Special Topics, 165:195-210, 2008.

[20] I. I. Rypina, M. G. Brown, F. J. Beron-Vera, H. Koçak, M. J. Olascoaga, and I. A. Udovydchenkov. Robust transport barriers resulting from strong kolmogorov-arnold-moser stability. Phys. Rev. Lett., 98(104102), 2007.

[21] D. Constantinescu and M.-C. Firpo. Integrability versus chaos in $11 / 2$ hamiltonian systems with broad perturbation spectrum. applications to the study of some transport phenomena. submitted for publication. 
[22] Ortega R. Invariant curves of mappings with averaged small twist. Advanced Nonlinear Studies, 1:14 - 39, 2001.

[23] C. D. Cothran, M. R. Brown, T. Gray, M. J. Schaffer, and G. Marklin. Observation of a helical self-organized state in a compact toroidal plasma. Phys. Rev. Lett., 103(21):215002, Nov 2009.

[24] J.H. Misguich, J.-D. Reuss, D. Constantinescu, G. Steinbrecher, M. Vlad, F. Spineanu, B. Weyssow, and R. Balescu. Noble internal transport barriers and radial subdiffusion of toroidal magnetic lines. Ann. Phys. Fr., 28(6):1-101, 2003.

[25] B. Weyssow I. Pavlenko, B. Rapoport and D. Carati. Hamiltonian mapping of magnetic reconnection during the crash stage of the sawtooth instability. Phys. Plasmas, 10:1083, 2003.

[26] S. S. Abdullaev. Construction of Mappings for Hamiltonian Systems and Their Applications. Springer, 2006.

[27] V. Igochine, O. Dumbrajs, D. Constantinescu, H. Zohm, G. Zvejnieks and the ASDEX Upgrade Team Nucl. Fusion, 46:741751, 2006.

[28] M. N Rosenbluth, R. Z Zagdeev, J. B. Taylor, and G. M. Zaslavski Destruction of magnetic surfaces by magnetic field irregularities. Nucl. Fusion, 6:297 - 300, Mar 1966.

[29] G. M. Zaslavsky, R. Z. Sagdeev, D. A. Usikov, and A. A. Chernikov. Weak Chaos and Quasi-Regular Patterns. Cambridge University Press, 1991.

[30] Following the definition given by G.M. Zaslavsky et al. in Ref. [29], the stochastic web is a thin net of fibers of finite width in the phase space of a Hamiltonian system with chaotic trajectories within the web and with regular dynamics outside it, at least in the web's vicinity. Its Hamiltonian prototype is fully degenerate, in the sense that $d^{2} H_{0} / d I^{2}$ is identically vanishing, with $H(I, \theta)=a I+\varepsilon H_{1}(I, \theta)$. The case considered, namely the dynamics of magnetic field lines for a magnetic winding profile in an action variable $I$ (that is equal to $\psi$ for a purely axi-symmetric equilibrium) is not fully degenerate in that $d^{2} H_{0} / d I^{2}$ vanishes only for some isolated $I$ value(s).

[31] A. A. Chernikov, R. Z. Sagdeev, D. A. Usikov, M. Yu Zakharov, and G. M. Zaslavsky. Minimal chaos and stochastic webs. Nature, 326:559-563, 1987.

[32] S. M. Soskin, O. M. Yevtushenko, and R. Mannella. Drastic facilitation of the onset of global chaos. Phys. Rev. Lett., 90:174101, 2003.

[33] J. P. van der Weele, and T. P. Valkering. The birth process of periodic orbits in non-twist maps. Physica A, 169:4272, 1990.

[34] J.E. Howard, S.M. Hohs. Stochasticity and reconnection in Hamiltonian systems. Phys. Rev. A, $29: 418421,1984$.

[35] D. del-Castillo-Negrete, and M.-C. Firpo. Coherent structures and self-consistent transport in a mean field Hamiltonian model. Chaos, 12:496507, 2002.

[36] P. J Morrison, and A. Wurm. Nontwist maps. Scholarpedia, 4(9):3551, 2009.

[37] A.A. Tuccillo et al. Development on jet advanced tokamak operations for iter. Nucl. Fusion, 46:214-224, 2006.

[38] O. Gruber, R. Arslanbekov, C. Atanasiu, A. Bard, G. Becker, W. Becker, M. Beckmann, K. Behler, K. Behringer, A. Bergmann, R. Bilato, D. Bolshukin, K. Borrass, H.-S. Bosch, B. Braams, M. Brambilla, R. Brandenburg, F. Braun, H. Brinkschulte, R. Brckner, B. Brsehaber, K. Bchl, A. Buhler, H. Brbaumer, A. Carlson, M. Ciric, G. Conway, D.P. Coster, C. Dorn, R. Drube, R. Dux, S. Egorov, W. Engelhardt, H.-U. Fahrbach, U. Fantz, H. Faugel, M. Foley, P. Franzen, P. Fu, J.C. Fuchs, J. Gafert, G. Gantenbein, O. Gehre, A. Geier, J. Gernhardt, E. Gubanka, A. Gude, S. Gnter, G. Haas, D. Hartmann, B. Heinemann, A. Herrmann, J. Hobirk, F. Hofmeister, H. Hohencker, L. Horton, L. Hu, D. Jacobi, M. Jakobi, F. Jenko, A. Kallenbach, O. Kardaun, M. Kaufmann, A. Kendl, J.-W. Kim, K. Kirov, R. Kochergov, H. Kollotzek, W. Kraus, K. Krieger, B. Kurzan, G. Kyriakakis, K. Lackner, P.T. Lang, R.S. Lang, M. Laux, L. Lengyel, F. Leuterer, A. Lorenz, H. Maier, K. Mank, M.-E. Manso, M. Maraschek, K.-F. Mast, P.J. McCarthy, D. Meisel, H. Meister, F. Meo, R. Merkel, V. Mertens, J.P. Meskat, R. Monk, H.W. Mller, M. Mnich, H. Murmann, G. Neu, R. Neu, J. Neuhauser, J.-M. Noterdaeme, I. Nunes, G. Pautasso, A.G. Peeters, G. Pereverzev, S. Pinches, E. Poli, R. Pugno, G. Raupp, T. Ribeiro, R. Riedl, S. Riondato, V. Rohde, H. Rhr, J. Roth, F. Ryter, H. Salzmann, W. Sandmann, S. Sarelma, S. Schade, H.-B. Schilling, D. Schlgl, K. Schmidtmann, R. Schneider, W. Schneider, G. Schramm, J. Schweinzer, S. Schweizer, B.D. Scott, U. Seidel, F. Serra, S. Sesnic, C. Sihler, A. Silva, A. Sips, E. Speth, A. Stbler, K.-H. Steuer, J. Stober, B. Streibl, E. Strumberger, W. Suttrop, A. Tabasso, A. Tanga, G. Tardini, C. Tichmann, W. Treutterer, M. Troppmann, N. Tsois, W. Ullrich, M. Ulrich, P. Varela, O. Vollmer, U. Wenzel, F. Wesner, R. Wolf, E. Wolfrum, R. Wunderlich, N. Xantopoulos, Q. Yu, M. Zarrabian, D. Zasche, T. Zehetbauer, H.-P. Zehrfeld, A. Zeiler, and H. Zohm. Overview of asdex upgrade results. Nuclear Fusion, 41(10):1369, 2001.

[39] M. Takechi, T. Fujita, Y. Ishii, T. Ozeki, A. Isayama, and the JT-60U Team. Mhd instabilities leading to disruptions in low beta jt-60u reversed shear plasmas. Nucl. Fusion, 45:1694-1699, 2005.

[40] G M D Hogeweij, Y Baranov, G D Conway, S R Cortes, M R De Baar, N Hawkes, F Imbeaux, X Litaudon, J Mailloux, F G Rimini, S E Sharapov, B C Stratton, K-D Zastrow, and contributors to the EFDA-JET workprogramme. Electron heated internal transport barriers in jet. Plasma Physics and Controlled Fusion, 44(7):1155, 2002.

[41] P. Maget, G. T. A. Huysmans, X. Garbet, M. Ottaviani, H. Lütjens, and J.-F. Luciani. Nonlinear magnetohydrodynamic simulation of tore supra hollow current profile discharges. Physics of Plasmas, 14(5):052509, 2007.

[42] S. Tokunaga, M. Yagi, S.-I. Itoh, and K. Itoh. Multi-scale transport simulation of toroidal momentum source profile effect on internal transport barrier collapse. Nuclear Fusion, 49:075023, 2009.

[43] M. R. de Baar, M. N. A. Beurskens, G. M. D. Hogeweij, and N. J. Lopes Cardozo. Tokamak plasmas with dominant electron cyclotron heating; evidence for electron thermal transport barriers. Physics of Plasmas, 6(12):4645-4657, 1999. 